\title{
Perfil dos Pacientes Portadores de Mielomeningocele na Cidade de Mogi das Cruzes
}

\author{
Profile of Patients with Myelomeningocele of Mogi das Cruzes City
}

\section{Alessandra Molteni Trautwein Bergamaschi ${ }^{1}$, Tereza Cristina Carbonari de Faria $^{2}$, Carlos Alberto dos Santos ${ }^{3}$}

\section{RESUMO}

A mielomeningocele (MMC) é uma malformação congênita frequente e ocorre entre a terceira e quinta semana de gestação. $\mathrm{O}$ objetivo deste trabalho foi verificar o perfil e o nível sócio-econômico dos pacientes na cidade de Mogi das Cruzes. Foram aplicados dois questionários aos pais de crianças com MMC, um direcionado à formação do perfil dos pacientes e outro para observar o nível sócio-econômico. Os resultados demonstraram que houve maior prevalência no sexo feminino, com maior incidência na regiáo lombar baixa. A maioria dos casos foi diagnosticado no período pré-natal e submetidos a correção nas primeiras 48 horas de nascimento. A hidrocefalia estava presente em todos os casos, sendo a troca da derivação ventrículo peritoneal realizada na metade deles. Todas as crianças tiveram infecção urinária, e a maioria deformidades ortopédicas, tendo o maior índice, o pé torto congênito. Dos casos em análise quase 50\% foram submetidos a cirurgias ortopédicas corretivas. Embora todos utilizassem órtese suropodálica, apenas $62,5 \%$ realizavam fisioterapia. A maioria estudava em escolas regulares e pertencentes da classe social C. Apenas $56,25 \%$ dos pais apresentavam conhecimento adequado sobre a patologia. Concluiu-se a grande importância do conhecimento do perfil dos pacientes para que os profissionais possam realizar de forma mais adequada seus tratamentos e orientaçóes.

Unitermos. Mielomeningocele, Perfil de Saúde, Qualidade de Vida, Reabilitação.

Citaçáo. Bergamaschi AMT, Faria TCC, Santos CA. Perfil dos Pacientes Portadores de Mielomeningocele na Cidade de Mogi das Cruzes.

\begin{abstract}
Myelomeningocele (MMC) is a congenital malformation and often occurs between the third and fifth week of gestation. The objective of this work was to verify the profile and socioeconomic level of the patients with MMC in Mogi das Cruzes. Two questionnaires were applied to parents of children with MMC, one seeking profile and the other the socioeconomic level of the patients. The results showed higher prevalence among females, with higher incidence in lower lumbar region. Most cases were diagnosed in the prenatal period and submitted to the correction within 48 hours of birth. Hydrocephalus was present in all cases, ventriculoperitoneal shunt was done in half of the cases. All children had urinary infection, the most presented orthopedic deformities, the majority have presented congenital clubfoot. Almost $50 \%$ of the analyzed cases performed surgery to repair it. Although all used ankle-foot orthoses, only $62.5 \%$ practiced physiotherapy. For the social economic and cultural aspect the most were studying in regular schools and socially they belong to class C. Only $56.25 \%$ of parents had good knowledge about the disease which victimize their children. It was concluded the great importance of knowing the patient profile so that professionals can perform more adequately their treatments and guidelines.
\end{abstract}

Keywords. Myelomeningocele, Health Profile, Quality of Life, Rehabilitation.

Citation. Bergamaschi AMT, Faria TCC, Santos CA. Profile of Patients with Myelomeningocele of Mogi das Cruzes City.
Trabalho realizado na Universidade de Mogi das Cruzes-SP, Brasil. 1.Fisioterapeuta, Clifisio- Clínica conveniada com a Santa Casa de Misericórdia de Suzano, Suzano-SP, Brasil.

2.Fisioterapeuta, Doutora, Professa na Universidade de Mogi das Cruzes (UMC), Mogi das Cruzes-SP, Brasil.

3.Fisioterapeuta, Mestre, Professor da Universidade Braz Cubas, Chefe da Fisioterapia do Hospital Auxiliar de Suzano da FMUSP, Suzano-SP, Brasil.
Endereço para correspondência:

Tereza Cristina Carbonari Faria Rua Adolfo Figueire do Rodrigues, 682 - Jardim Aracy CEP 08779-555, Mogi das Cruzes-SP, Brasil.

E-mail: t.faria@uol.com.br 


\section{INTRODUÇÃO}

A mielomeningocele (MMC) é a forma mais frequente de um grupo de doenças denominadas defeitos de fechamento do tubo neural (DFTN). A falha na fusão dos elementos posteriores da coluna vertebral produz malformação das lâminas e processos espinhosos do canal vertebral e também displasia da medula espinhal ${ }^{1,2}$.

Esta patologia pode ser diagnosticada no período pré-natal pela ultrassonografia a partir da $16^{\mathrm{a}}$ semana de gestação, sendo certo que tais aparelhos possibilitam com chance de 80 a $90 \%$ de sucesso na detecção nestes exames, porém, a precisáo e a qualidade do mesmo vai depender da experiência do examinador, do equipamento e do tempo dedicado a cada exame $e^{3,4}$.

A etiologia é desconhecida, mas apresenta características multifatoriais (genéticas e ambientais). Dentre os fatores ambientais, sabe-se que mulheres com dieta pobre em ácido fólico (vitamina B9) possuem maior chance de terem filhos afetados pela doença ${ }^{5}$.

No período de 1967 a 1995, a prevalência observada pelo Estudo Colaborativo Latino-Americano de Malformaçóes Congênitas (ECLAMC), sobre 4 milhôes de nascimentos na América Latina, foi de 1,5:1.000. Quanto à prevalência, no Brasil, dados mostram que varia de 0,83: 1.000 a 1,87: 1.000 nascidos vivos e incidência de 1,8:1000 nascimentos ${ }^{5,6}$. Já em um estudo realizado na Universidade de Campinas, entre 1982 e 2001, apontou uma incidência de 2,28:1.000 nascimentos ${ }^{3,6,7,8}$.

Dentre as patologias que acompanham a MMC, a mais frequente é a hidrocefalia congênita, que ocorre pela alteração da drenagem de líquor devido a protrusáo do tecido nervoso central, que leva a herniação do tronco cerebral e do assoalho do quarto ventrículo para o canal cervical medular e o deslizamento do cerebelo pelo forame magno. Essa complicação requer a necessidade de procedimentos cirúrgicos para derivação liquórica e controle da pressão intracraniana ${ }^{3}$.

O portador de MMC pode ainda apresentar outras anomalias como as deformidades esqueléticas, sendo as mais observadas, a presença de pé torto e a luxação de quadril ao nascimento e malformaçóes da coluna vertebral como as hipercifoses e as escolioses?

$\mathrm{O}$ tratamento padrão da $\mathrm{MMC}$ tem sido a correção cirúrgica neonatal, e o fechamento deve ser feito den- tro das 48 horas após o nascimento, dessa forma sendo possível reduzir os riscos e infecçóes ${ }^{10}$.

Quanto ao nível funcional dos indivíduos com MMC a classificação mais utilizada é a de Hoffer que classifica a MMC de acordo com o comprometimento neurológico em quatro níveis. No nível torácico, o paciente não apresenta movimentação ativa nos membros inferiores; no lombar alto, apresenta funcionantes os músculos psoas, adutores e eventualmente o quadríceps; no lombar baixo, encontra funcionantes o psoas, adutores, quadríceps, flexores mediais do joelho e eventualmente tibial anterior e/ou glúteo médio; por fim, o nível sacral apresenta funcionantes os músculos acima citados, mais a função flexora plantar e/ou extensora do quadril ${ }^{5}$.

O nível anatômico da lesão é fator essencial que influencia o prognóstico de deambulação em crianças com MMC. Os fatores adicionais são as deformidades músculoesqueléticas, outras deficiências neurológicas como a do neurônio motor superior, sinais e sintomas de malformações intraespinhal, bem como o gasto energético e tipo de órtese ${ }^{11}$.

A importância de se conhecer o perfil dos pacientes de MMC é essencial para que o tratamento e as técnicas sejam direcionados a esses pacientes, aproximando a fisioterapia da realidade da população a qual presta serviços. Esse envolvimento entre terapeutas, familiares e crianças, tem obtido resultados positivos no desenvolvimento das mesmas, contribuindo para que tenham um melhor prognóstico de independência ${ }^{12}$.

Sendo assim este trabalho teve como objetivo traçar um perfil clínico dos pacientes portadores de MMC na cidade de Mogi das Cruzes; verificar o nível de conhecimento dos pais sobre esta patologia e as suas condiçóes sociais.

\section{MÉTODO}

\section{Amostra}

Participaram desta pesquisa 16 pais de portadores de MMC, credenciados na APAE de Mogi das Cruzes, Clínica de Fisioterapia da Faculdade Náutico Mogiano e Clínica de Fisioterapia da Universidade de Mogi das Cruzes sem critérios de idade e gênero. Após a aprovação do projeto de pesquisa pelo Comitê de Ética em Pesquisa da Universidade de Mogi das Cruzes (CAAE: 0087237.000- 
09), os participantes assinaram um termo de consentimento livre e esclarecido.

\section{Procedimento}

Para a coleta de dados, foram aplicados dois questionários. Um direcionado para avaliação clínica dos pacientes pelo fisioterapeuta, através da anamnese e exame físico, e outro para critério de classificação econômica da Associação Brasileira de Empresas de Pesquisa (ABEP). Os questionários foram aplicados individualmente aos pais, nas Instituiçôes citadas acima, com esclarecimento momentâneo das dúvidas apresentadas pelos mesmos.

\section{Análise estatística}

Os dados foram tabulados e a análise realizada a partir de porcentagens simples de regra de três.

\section{RESULTADOS}

Os resultados demonstraram que $75 \%$ dos portadores de MMC eram do sexo feminino e $25 \%$ do masculino. A região anatômica mais acometida foi a regiâo lombar baixa com 12 casos (75\%), seguida da lombar alta com 3 casos $(18,75 \%)$ e região torácica com 1 caso $(6,25 \%)$.

Foram diagnosticados 12 casos no período prénatal $(75 \%)$ e 4 casos no peri-natal (25\%), sendo que $75 \%$ destes, foram submetidos a correção cirúrgica nas primeiras 48 horas de nascimento.

Embora todas as crianças apresentassem hidrocefalia, apenas $2(12,5 \%)$ não necessitaram de colocação da DVP. Das $14(87,5 \%)$ que receberam a válvula, 8 casos $(57,14 \%)$ já havia realizado sua troca. E todos os casos (100\%) tiveram uma ou mais infecções urinárias.

Quatorze crianças $(87,5 \%)$ possuíam uma ou mais deformidades musculoesqueléticas, sendo a mais encontrada, o pé torto congênito em 12 (75\%), seguido pela escoliose em 4 casos $(23,53 \%)$ e luxação de quadril em apenas 1 caso $(5,88 \%)$. Nove delas $(64,29 \%)$, passaram por cirurgias para correção. Ainda que todas as crianças fizessem uso de aparelhos ortopédicos, apenas 10 delas $(62,5 \%)$ realizavam fisioterapia.

Referente à participação escolar, 10 casos (62,5\%) frequentavam escolas de ensino regular, 3 casos $(18,75 \%)$ especiais e 3 casos $(18,75 \%)$ não estudavam. Quanto a classe sócio-econômica, 2 casos (12,5\%) pertenciam a classe B2, 7 casos $(43,75 \%)$ a C1, 6 casos $(37,5 \%)$ a C2 e apenas 1 caso $(6,25 \%)$ a D. Observou-se que 9 pais $(56,25 \%)$ sabiam da patologia dos seus filhos, 4 pais $(25 \%)$ sabiam, mas apresentavam dúvidas e 3 pais $(18,75 \%)$ não sabiam.

\section{DISCUSSÃO}

Os achados desta pesquisa demonstraram predominância do sexo feminino em portadores de MMC, como relatado na literatura ${ }^{7,13}$.

O mesmo ocorreu em relação às regióes acometidas, onde, no presente estudo, observou-se maior acometimento na regiáo lombar baixa, seguida da alta e torácica, não apresentando na região sacral, o que se assemelhou com outros autores, que demonstraram a mesma sequência de incidência, porém, com acometimento de 3\% na região sacral ${ }^{3}$.

Através da ultrassonografia é possível diagnosticar a espinha bífida a partir do terceiro mês de gestação, mostrando a importância deste exame para um diagnóstico precoce e intervenção planejada no período peri-natal, devendo a mesma ser feita de 24 a 48 horas de vida, prevenindo-se assim a possibilidade de maiores infecçóes do sistema nervoso central, como a ventriculite e meningi$\mathrm{te}^{14}$. No presente estudo, apesar de mais da metade ter sido diagnosticado no período pré-natal, 25\% fizeram a correção cirúrgica somente após as 48 horas de vida.

A hidrocefalia é uma complicação frequente, estando presente em cerca de $90 \%$ dos casos de $\mathrm{MMC}^{13}$. O tratamento utilizado para esta complicação é a cirurgia de DVP, sendo que a maioria destes pacientes realizará, ao menos, uma derivação nos primeiros meses de vida. Alguns necessitarão de revisóes das válvulas durante o crescimento, já que complicações são frequentes nesse procedimento $^{15,16}$. Os resultados adquiridos nessa pesquisa demonstraram que a mesma estava presente em todos os casos, com necessidade da colocação da DVP em 87,5\%, sendo sua troca já realizada em $57,14 \%$ dos casos.

Outra complicação que pode ser observada em crianças com MMC, é a infecção urinária, decorrente geralmente de bexiga neurogênica, que leva a retenção urinária. Tal complicação constitui grande ameaça à sobrevivência da criança, ficando atrás apenas da hidroce- 
falia ${ }^{17}$. Neste estudo, todos os casos tiveram um ou mais episódios de infecção urinária, demonstrando a necessidade de observaçóes frequentes e intervençóes precoces, para diminuir o sofrimento e a ameaça à vida da criança.

Quanto às deformidades, houve predomínio de pé torto congênito, seguido de escoliose e luxação de quadril. Na literatura os resultados foram semelhantes, porém, a luxação de quadril teve maior predominância em relação às alteraçóes da coluna vertebral ${ }^{9}$.

Em outro estudo os autores citaram, que dos 53 participantes portadores de $\mathrm{MMC}$, apenas um, não utilizava órtese, o que corresponde aos encontrados no presente estudo, em que todos os participantes faziam uso de uma ou mais órteses ${ }^{18}$. Todos os casos passaram por tratamento fisioterapêutico, sendo que apenas $62,5 \%$ continuavam a realizar o mesmo.

Apesar de ser uma malformação congênita, quase $50 \%$ dos pais apresentaram dúvidas ou não sabiam da patologia, sendo que estas crianças apresentavam idade entre 1 e 12 anos de idade, o que demonstra o déficit de informaçóes aos pais pela equipe de saúde. Segundo a literatura, é necessária maior observação na orientação dada aos cuidadores, principalmente por terem grande influência no desenvolvimento destas crianças e que a estrutura familiar e o nível de educação dos pais determinam um cuidador mais preparado ${ }^{19}$. Tal observação também foi citada por outros autores, ressaltando que a família é de grande valor para o aprendizado das habilidades básicas, o que é primordial para o alcance de uma maior independência ${ }^{12}$.

Ainda nos dias de hoje há muitos obstáculos na interação entre crianças com deficiência como a MMC e a sua participação na sociedade, devido às barreiras existentes. Esse ajuste psicossocial é de grande valor para se obter bons resultados, desta forma favorecendo a inclusão social $^{12}$. Nos resultados encontrados, observou-se que a maioria em idade escolar frequentavam escolas, seja do ensino regular ou especial, sendo que apenas uma das crianças não frequentava, devido à baixa cognição. Tal fato demonstrou a interação social destas crianças.

A disponibilidade de recursos financeiros familiares também afeta a qualidade de vida dos portadores de deficiências, diminuindo o contato com os tratamentos apropriados e os recursos disponíveis ${ }^{18}$. Nesse estudo, a maioria $(81,25 \%)$ era da classe $\mathrm{C}$, fato este que representa condição humilde de vida, principalmente em termos de aquisição de bens, como as órteses, nos casos de MMC.

Conclui-se que após a coleta de dados, foi traçado o perfil dos pacientes com MMC da cidade de Mogi das Cruzes, assim como o nível de conhecimento dos pais sobre esta patologia e a classe sócio-econômica a que pertenciam.

Observou-se que a MMC é uma malformação muito complexa, sendo necessário um melhor conhecimento dos perfis dos portadores da mesma, para que os fisioterapeutas possam oferecer orientações mais adequadas a essas famílias, melhorando de alguma forma suas condições de vida. Esta adequação paciente/família e fisioterapeuta é primordial para o sucesso dos tratamentos.

Como sugestão há necessidade da realização de estudos similares, com maior número de casos para que cada vez mais possamos adequar as terapias à realidade de vida de nossos pacientes.

\section{REFERÊNCIAS}

1.Fernandes AC, Saito ET, Faria JC, Zuccon A. Aspectos Clínicos e Práticos da Reabilitaçấo. São Paulo: Artes Médicas Ltda; 2007, p.87-97.

2.Aguiar MJB, Campos AS, Aguiar RALP, Lana MAS, Magalhães RL, Babeto LT. Defeitos de fechamento do tubo neural e fatores associados em recémnascidos vivos e natimortos. J pediatr 2003;79:129-33.

http://dx.doi.org/10.2223/JPED.964

3.Souza ASR, Arruda TAM, Amorim MMA, Pacheco AJC. Espinha Bífida: Conceito atuais. Rev Feminina 2007;35:455-62.

4.Hisaba WJ, Moron AF, Cavalheiro S, Santana RM, Passos JP, Cordioli E. Espinha bífida aberta: achados ultra-sonográficos e presença de contraçóes uterinas na predição da evolução motora neonatal. Rev Bras Ginecol Obstet 2003;25:425-30. http://dx.doi.org/10.1590/S0100-72032003000600007

5.Rocco FM, Saito ET, Fernandes, AC. Perfil dos pacientes com Mielomeningocele da Associação de Assistência à Criança Deficiente (AACD). Rev Acta Fisiátrica 2007;14:130-3.

6.Grillo E, Silva RJM. Defeitos do tubo neural e hidrocefalia congênita. Por que conhecer suas prevalências? J pediatr 2003;79:105-6.

http://dx.doi.org/10.2223/JPED.960

7.Madeira L. Mielomeningocele: Desfechos Neurocirúrgicos no Hospital Infantil Joana de Gusmão [trabalho de conclusão de curso]. Florianópolis: Universidade Federal de Santa Catarina; 2007, p.1-29.

8.Sbragia L. Evolução de 58 fetos com meningomielocele e o potencial de reparo intra-útero. Arq Neuro-psiquiatria 2004;62:487-491.

http://dx.doi.org/10.1590/S0004-282X2004000300020 
9.Christofoletti G. Alteraçôes Motoras e Sensoriais na Mielomeningocele: Relato de Três Casos. Saúde em Revista, 2007;22:53-7.

10.Ulsenheimer MMM, Antoniuk AS, Santos LHC, Ceccatto MP, Silveira AE, Ruiz AP, et al. Myelomeningocele: A Brazilizn University Hospital Experience. Arq Neuro-psiquiatria 2004;62:963-8.

http://dx.doi.org/10.1590/S0004-282X2004000600006

11.Bartonek $\AA$, Saraste H. Factors influencing ambulation in myelomeningocele: a cross-sectional study. Dev med child neurol 2001;43:253-60.

http://dx.doi.org/10.1017/S0012162201000482

http://dx.doi.org/10.1111/j.1469-8749.2001.tb00199.x

12.Vachha, B, Admas R. Implications of family environment and language development: comparing typically developing children to those with spina bifida. Pediatric Developmental Disabilities, Texas Scottish Rite Hospital for Children, and Behavioral and Brain Sciences. University of Texas at Dallas, Dallas (USA); 2009, p.709-716.

13.Pereira ELR, Bichara CNC, Oliveira ACS, Costa MAT. Epidemiologia de Pacientes com Malformação de Chiari II Internados no Hospital Fundação Santa Casa de Misericórdia do Pará. Rev Para Med 2007;21:13-7.
14.Salomão JF, Pinheiro JAB, Carvalho JGS, Leibinger RD, Lucchesi G, Bonfim V. Mielomeningocele: Tratamento cirúrgico e resultados. J Pediatr 1995:71:321-7.

15.Martínez-lage JF, Péreza-espejo MA, Almagro MJ, López F, Piqueras C, Tortosa J. Síndromes de hiperdrenaje de las válvulas en hidrocefalia infantil. Neurocir 2005;16:124-33.

16.Faria TCC. Avaliação Neuromotora em pacientes portadores de mielomeningocele submetidos à intervenção cirúrgica intrauterina [tese]. São Paulo: Universidade Federal de São Paulo; 2009, p.18-22.

17.Shepherd R. Fisioterapia em Pediatria. 3a ed. São Paulo (SP): Santos; 1998, p. $95-102$

18.Cate IMPN, Kennedy C, Stevenson J. Disability and quality of life in spina bifida and hydrocephalus. Dev med child Neurol 2002;44:317-22.

http://dx.doi.org/10.1111/j.1469-8749.2002.tb00818.x

19.Kirpalani HM, Parkin PC, Willian AR, Fehlings DL, Rosenbaum PL, King $\mathrm{D}$, et al. Quality of life in spina bifida: importance of parental hope. Arch Dis Child 2000;83:293-7.

http://dx.doi.org/10.1136/adc.83.4.293 